\title{
How can interventions increase motivation for physical activity? A systematic review and meta- analysis
}

Keegan Knittle, Johanna Nurmi, Rik Crutzen, Nelli Hankonen, Marguerite Beattie \& Stephan U. Dombrowski

To cite this article: Keegan Knittle, Johanna Nurmi, Rik Crutzen, Nelli Hankonen, Marguerite Beattie \& Stephan U. Dombrowski (2018): How can interventions increase motivation for physical activity? A systematic review and meta-analysis, Health Psychology Review, DOI: 10.1080/17437199.2018.1435299

To link to this article: https://doi.org/10.1080/17437199.2018.1435299

Accepted author version posted online: 31 Jan 2018.

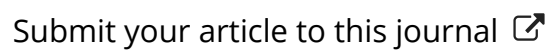

Llll Article views: 72

Q View related articles $₫$

View Crossmark data $\nearrow$ 
Publisher: Taylor \& Francis \& Informa UK Limited, trading as Taylor \& Francis Group Journal: Health Psychology Review

DOI: $10.1080 / 17437199.2018 .1435299$

\section{How can interventions increase motivation for physical activity?}

\section{A systematic review and meta-analysis}

Keegan Knittle (1*), Johanna Nurmi (1,2), Rik Crutzen (3),

Nelli Hankonen (1,4), Marguerite Beattie (1), \& Stephan U Dombrowski (5)

1. Department of Social Research - Social Psychology; P.O. Box 54; 00014 University of Helsinki, Finland; Phone: +358 (0)504487787; Email: keegan.knittle@helsinki.fi

2. Behavioural Science Group, Institute of Public Health, University of Cambridge, Forvie Site, Robinson Way, Cambridge, CB2 0SR, UK; Phone: +358 (0)503421436; Email: johanna.nurmi@helsinki.fi

3. Department of Health Promotion, Maastricht University/CAPHRI, P.O. Box 616, 6200 MD Maastricht, The Netherlands; Phone: +31 (0)433882828; Email: rik.crutzen@maastrichtuniversity.nl

4. Faculty of Social Sciences, University of Tampere / Linna, 33014 Tampere, Finland Phone: +358 (0); Email.nelli.hankonen@staff.uta.fi

5. Faculty of Natural Sciences, Division of Psychology, University of Stirling, UK; Phone: +44 (0)1786467844; Email: s.u.dombrowski@stir.ac.uk

* - Corresponding author.

\section{Funding sources}

This review was partially funded by a grant to Keegan Knittle from the Netherlands

Organization for Scientific Research (NWO project \#: 446-14-004) and by grants to Nelli Hankonen from the Academy of Finland (funding numbers 295765 (KK) and 285283 (NH)).

\section{Acknowledgments}


Thank you to Matthias Aulbach for statistical assistance with moving constant analyses, to Mirte Reimerink for her help with creating tables, and to the authors of included studies who responded to our requests for additional data or information. 


\begin{abstract}
Motivation is a proximal determinant of behavior, and increasing motivation is central
\end{abstract} to most health behavior change interventions. This systematic review and meta-analysis sought to identify features of physical activity interventions associated with favorable changes in three prominent motivational constructs: intention, stage of change and autonomous motivation. A systematic literature search identified 89 intervention studies $(k=200 ; N=19,212)$ which assessed changes in these motivational constructs for physical activity. Intervention descriptions were coded for potential moderators, including behavior change techniques (BCTs), modes of delivery and theory use. Random effects comparative subgroup analyses identified 18 BCTs and 10 modes of delivery independently associated with changes in at least one motivational outcome (effect sizes ranged from $d=0.12$ to $d=0.74)$. Interventions delivered face-to-face or in gym settings, or which included the BCTs 'behavioral goal setting', 'self-monitoring (behavior)' or 'behavioral practice/rehearsal', or which combined self-monitoring (behavior) with any other BCT derived from control theory, were all associated with beneficial changes in multiple motivational constructs (effect sizes ranged from $d=0.12$ to $d=0.46$ ). Meta-regression analyses indicated that increases in intention and stage of change, but not autonomous motivation, were significantly related to increases in physical activity. The intervention characteristics associated with changes in motivation seemed to form clusters related to behavioral experience and self-regulation, which have previously been linked to changes in physical activity behavior. These BCTs and modes of delivery merit further systematic study, and can be used as a foundation for improving interventions targeting increases in motivation for physical activity.

Keywords: Meta-analysis; physical activity; intention; stage of change; autonomous motivation; behavior change techniques 
Review Registration: This study was pre-registered in PROSPERO, the international prospective register of systematic reviews (ID\#: $\underline{\text { CRD42015014922) }}$

All supplementary materials are available on Open Science Framework at https://osf.io/2fqr3/ 
How can interventions increase motivation for physical activity?

A systematic review and meta-analysis

Physical inactivity is strongly associated with premature mortality and the development of cardiovascular and metabolic diseases (Matthews et al., 2012), and presents considerable financial costs to society (Ding et al., 2016). As a result, governments have begun to prioritize population-level participation in physical activity to prevent the costs associated with rising rates of lifestyle-related illnesses.

These prevention efforts rely on interventions which effectively increase physical activity, and physical activity interventions have been developed and tested across a range of settings and populations with varying success. Previous meta-analyses indicate that, cumulatively, behavioral interventions produce significant small-to-medium changes in both subjectively- and objectively-measured physical activity (Hobbs et al., 2013; Olander et al., 2013). However, within-studies, there is evidence that these interventions do not lead to increases in physical activity for all individuals (Adams \& White, 2005; Harrison, Roberts \& Elton, 2005). Meta-analyses have also given some indications of the factors of interventions associated with more effective interventions, including the inclusion of behavior change techniques (BCTs) derived from control theory (Carver \& Scheier, 1982) and BCTs targeting social support (Olander et al., 2013).

Increasing motivation, defined as "a driving force or forces responsible for the initiation, persistence, direction, and vigor of goal-directed behavior" (Oxford dictionary of psychology, 2014), is a central ambition of many programs designed to increase physical activity (Schwarzer, Lippke \& Lusczynska, 2011). Motivation not only determines whether individuals will make efforts to change their physical activity behavior in the first place, but also whether they will take up or engage with action-focused (e.g. self-regulatory) components of interventions (McMurran \& Ward, 2010; Schwarzer et al., 2011), and whether 
newly-enacted behavioral changes are likely to be maintained in the long term (Kwasnicka, Dombrowski, White \& Sniehotta, 2016). Motivation may also be a key explanatory factor of socioeconomic differences in physical activity (Hankonen et al., 2017). An incomplete understanding of how to increase motivation results in an incomplete understanding of how to increase physical activity itself, but to date, experimental and meta-analytical research on physical activity interventions has focused primarily on behavioral outcomes. A more complete understanding of how interventions can increase motivation is therefore key to fully understanding the psychological process of physical activity behavior change and to developing effective physical activity interventions.

\section{How is Motivation Conceptualized within Behavioral Theories?}

Nearly all behavioral theories propose a hierarchy in which social-cognitive and environmental factors predict some seminal motivational construct, which triggers (or is closely aligned with) a shift from motivation to behavioral enactment. Crossing this 'decisional Rubicon' (Gollwitzer, 1990) from the motivational or pre-intentional phase into the post-intentional, volitional, or action phase rarely occurs spontaneously, and motivational constructs and their corresponding theoretical determinants have been conceptualized differently across theories. Three prominent theoretical conceptualizations of motivation are intention, stage of change, and autonomous motivation.

Behavioral intention. Numerous theories, such as the theory of planned behavior (Ajzen, 1991), reasoned action approach (Fishbein \& Ajzen, 2011) and health action process approach (Schwarzer et al., 2011), place intention, which indicates an individual's desire to perform a given behavior (Ajzen, 2002), as the proximal determinant of behavior separating motivation and action. Within the reasoned action approach, intention is predicted by individuals' attitudes, subjective norms and perceived behavioral control (which includes 
self-efficacy) toward the behavior (Ajzen, 1991; Fishbein \& Ajzen, 2011), which are in turn predicted by past behavior and various background variables (e.g. personality).

Several routes to forming and strengthening intention have been proposed in the literature, which include direct routes, such as identifying discrepancies between current and desired states and setting goals to narrow the discrepancy, with defining the goal itself roughly equivalent to intention formation (Maes \& Karoly, 2005), and more indirect routes that operate through theoretical determinants of intention or behavior. Examples of indirect routes to intention formation include information provision to induce fear or susceptibility (Ruiter, Abraham \& Kok, 2001), positive first- or second-hand experiences with the behavior to increase self-efficacy (Ashford, Edmunds \& French, 2010), and social support for the behavior to alter subjective norms (Hagger et al., 2009). Meta-analyses have revealed several additional BCTs which may lead to the formation of physical activity intentions via increases in self-efficacy (i.e. one's belief in his or her abilities to undertake a behavior) (Bandura, 1977), including behavioral feedback, providing instruction, action planning and reinforcement schedules or rewards (Williams \& French, 2011; Ashford et al., 2010), as well as verbal persuasion about capability (Steinmetz, Knappstein, Ajzen, Schmidt \& Kabst, 2016).

Previous meta-analyses indicate that interventions have positive small-to-medium cumulative effects on intention for physical activity (Steinmetz et al., 2016; Rhodes \& Dickau, 2012). However, despite the predominance of intention in several theories, no studies have yet investigated which intervention components or BCTs are most effective in increasing intention for physical activity. Identifying effective methods to strengthen intentions for physical activity may therefore improve the efficacy of physical activity interventions and contribute to renewal or further development of these theories. 
Stage of change and the transtheoretical model. While many social-cognitive theories are regarded as continuum models of behavior, the transtheoretical model (Prochaska \& DiClemente, 1986) is a stage theory, which assumes that individuals move through multiple distinct "stages of change" on their journey to adopting and maintaining a behavior. The stages of change (usually five, but sometimes extended to six or more) range from precontemplation, wherein a person has not considered changing their behavior, through to maintenance, where a person has successfully adopted a new behavior for at least six months and works to prevent relapsing into old patterns of behavior.

Within the transtheoretical model, cognitive, affective and behavioral "processes of change" are hypothesized to facilitate stage transitions (Prochaska \& Velicer, 1997), although there is some evidence that these are less applicable to physical activity than to other behaviors (Marshall and Biddle, 2001). For example, consciousness raising (i.e. gathering information about the behavior in question) and dramatic relief (i.e. introspection about feelings related to the behavior) should facilitate the transition from precontemplation to contemplation, but would not be expected to foster transitions from preparation to action or from action to maintenance (Prochaska \& Velicer, 1997).

Only one process of change, self-liberation, is hypothesized to help individuals transition from the preparation stage, in which intentions are formed and strengthened, into the action stage, in which individuals have taken considerable steps toward full adoption of the new behavior. Self-liberation includes individuals' examining their beliefs that change is possible and making commitments to act on those beliefs, and as such, parallels have been drawn between self-liberation and elements of both self-efficacy and intention from the theory of planned behavior (Armitage, 2009). Additionally, self-liberation resembles the BCT 'commitment' from the v1 taxonomy (Michie et al, 2013), in which individuals reaffirm their commitments to behavior change. 
While intention formation is hypothesized to occur in the preparation stage, the transtheoretical model does not clearly propose methods for assessing variance in intention strength. Studies using the transtheoretical model have instead relied on examining transitions between stages or perceived pros and cons of changing (i.e. decisional balance) to assess motivation. Although a vast body of empirical and experimental research based on the transtheoretical model exists, these findings have not yet been compiled meta-analytically to identify the BCTs most influential in phase transition.

Autonomous motivation and self-determination theory. Self-determination theory (Deci \& Ryan, 2000) proposes several sub-categories of motivation, which can be situated on a spectrum ranging from autonomous motives to controlled motives. On one side of this spectrum is intrinsic motivation, which is fully autonomous, and is characterized by the inherent satisfaction and pleasure gained from engaging in a behavior (Ryan \& Deci, 2000). Beyond intrinsic motivation lie extrinsic motivations, which are further classified by the degree to which they are internalized (Ryan \& Connell, 1989): from integrated (most autonomous) on the one hand, to external (most controlling) on the other.

Autonomous motivation is characterized by a sense of choice, volition, and freedom from external pressure to engage in the behavior, and consists of intrinsic motivation and two types of external motivation: integrated and identified. In other words, motivation is autonomous when it is engaged in for pleasure or fun (intrinsic motivation), when it is congruent with an individual's sense of self (integrated regulation), or when it is personally important to the individual (identified regulation).

Autonomous motivation is associated with positive changes in physical activity and other health behaviors (Hagger \& Chatzisarantis, 2009; Teixeira et al., 2012), as well as longterm maintenance of physical activity (Ng et al., 2012; Knittle, De Gucht, Hurkmans, Vliet Vlieland \& Maes, 2016). Controlled motivations, on the contrary, include external regulation 
(in which behavior is enacted to obtain a reward or avoid punishment) and introjected regulation (in which behavior is enacted to avoid guilt) (Deci \& Ryan, 2000), and are associated with less behavioral maintenance and poorer psychological well-being ( $\mathrm{Ng}$ et al., 2012).

Self-determination theory suggests that the internalization of behavioral regulation may be achieved by supporting individuals' needs for autonomy, competence, and relatedness (Ryan, 1995). This could include offering meaningful rationales for behavior or choices for behavioral enactment, using autonomy-supportive language, recognizing individuals' efforts, and fostering positive interactions with others - techniques which are closely aligned with principles of motivational interviewing (MI; Markland, Ryan, Tobin \& Rollnick, 2005) and have been theorized to increase autonomous motivation for physical activity (Markland \& Vansteenkiste, 2007). No previous meta-analyses have brought together empirical findings to identify the optimal methods to improve autonomous motivation for physical activity, which could contribute to better initiation and maintenance of behavior within interventions.

\section{Aims of the Present Review}

Physical activity interventions often draw from the theories presented above and target improvements in motivational variables en route to changing behavior. Understanding how to optimally foster changes in motivation for physical activity will help to improve behavioral theories in this domain and improve the capabilities of future interventions to motivate individuals to take up and maintain active lifestyles. This systematic review and meta-analysis primarily aims to identify BCTs, which, when included in physical activity interventions, are associated with changes in prominent measures of motivation: intention, stage of change and autonomous motivation. In addition, as additional study- and intervention-related factors can moderate intervention effects on motivation, this study will 
examine the extents to which modes of delivery, theory use, and participant characteristics are associated with changes in motivational outcomes for physical activity. Finally, this metaanalysis will examine the extents to which the effects of interventions on intention, stage of change and autonomous motivation predict the effects of interventions on physical activity behavior.

\section{Methods}

This systematic review and meta-analysis was prospectively registered in the PROSPERO register of systematic reviews (Knittle et al., 2015).

\section{Study Identification}

Literature searches were conducted in PsycInfo, Web of Science, PubMed and Google Scholar using the comprehensive search strategy available in the appendix. The search strategy was purposefully broad enough to capture any study which might have assessed physical activity, and therefore potentially also some measure of motivation for physical activity. A request for data from unpublished intervention studies was sent to members of the European Health Psychology Society. The final searches were conducted in February 2016.

To be eligible for inclusion, a study must have described an intervention delivered to adults and reported data on a measure of intention to be physically active, stage of change for physical activity or autonomous motivation for physical activity for at least two time points (i.e. just before the start of the intervention plus one other), so that pre-treatment to posttreatment changes in that variable could be assessed. Furthermore, study data needed to allow for the calculation of effect sizes, either from the article itself, supplementary material or after requests to the corresponding author(s). No further restrictions were placed on the types of interventions, study designs or participants. Studies were excluded if they did not meet the inclusion criteria, or if the first measurement point after baseline took place more than 26 
weeks after the intervention started, as we were interested in examining changes in motivation in the early phases of physical activity behavior change. We also excluded studies which reported on intention to increase physical activity, as changes in this measure would be confounded by any contemporaneous changes in physical activity behavior. Measures of motivation could be assessed in relation to any form of physical activity, which is defined as "any bodily movement produced by skeletal muscles that results in energy expenditure" (Caspersen, Powell \& Christenson, 1985, p. 126).

After conducting database searches, one researcher (KK) screened the titles and abstracts of retrieved records and eliminated duplicates and articles that certainly did not meet the inclusion criteria (e.g. animal studies, studies in children, studies in research domains not related to health or behavior change). At this stage, exclusions were only made in cases where it was certain that the record did not meet the inclusion criteria (e.g. not an intervention study, no mention of any outcome related motivation, physical activity, or energy balance-related outcomes like weight loss). For all articles not excluded after title and abstract screening, we sought full-text reports to determine eligibility for inclusion.

After obtaining the full texts of articles, we established the reliability of identifying eligible studies within our research group in a two-step process. First a random selection of 10 full text articles was screened by all researchers, and decisions on inclusion/exclusion were discussed within the group. Second, after jointly screening a second round of 10 full text articles, we reached full consensus on inclusion/exclusion, and subsequently proceeded with single-author screening.

For the remaining full text articles, one researcher (KK) independently reviewed each against the inclusion criteria. In situations when it was not clear whether a study fulfilled the inclusion criteria and contained appropriate outcome data, the full-text was also reviewed by a second randomly-assigned researcher, and discussions took place within the study team 
until a consensus decision was reached. Where a study fulfilled all inclusion criteria but presented data in a way that was unsuitable for meta-analysis, the corresponding authors were contacted by phone, email or through scientific social networks (e.g. ResearchGate, LinkedIn) to obtain additional data.

\section{Coding and Data Extraction}

After identifying the final set of included studies, we coded all study arms for the following moderator variables: BCT use (using the v1 taxonomy of BCTs [Michie et al., 2013]); sample characteristics (age, gender, healthy/risk/disease group, BMI/overweight status, recruitment method, setting, existing level of physical activity, socioeconomic status, education, income level); intervention characteristics (interyention label, group/individual, whether it included components delivered through digital, mobile, face-to-face, paper-based, SMS, phone or email channels, the total contact time, number of contacts, interventionist, theoretical basis (using item five from the theory coding scheme of Michie \& Prestwich, [2010]); and study characteristics (country, year, total length of follow-up, timing of measurements and the measurement instruments used for assessing outcomes). In accordance with the Iterative Protocol for Evidence Base Accumulation (Peters, De Bruin \& Crutzen, 2015), control group BCT content was coded independently from intervention group BCT content to isolate the 'active ingredients' being tested within each arm. Coding all study arms, as opposed to only active treatment arms, allows for more insights into how intervention content relates to outcomes (Peters et al., 2015)

To ensure consistency in applying the coding scheme, a random selection of five studies was pilot-coded by all researchers independently (KK, JN, NH, RC, and SD), and inter-rater reliability was calculated and checked against existing standards (Landis \& Koch, 1977). All discrepancies in this pilot-coding were then discussed within the study team to reach consensus, and where applicable, decision rules were created to inform coding and 
discussions of subsequent studies. Potential BCTs identified in treatment descriptions of the included studies that did not match with any of the BCTs listed in the v1 taxonomy were discussed within the entire study group and added as supplements to the taxonomy following the procedures reported elsewhere (Henrich et al., 2015). Pilot-coding continued in this way (five studies at a time, coded by all coders) for two rounds, until inter-rater reliability reached an acceptable level of $k=0.70$ for all coded moderators (Landis \& Koch, 1977). The remaining studies were independently coded by one researcher and checked by a second researcher selected at random using Microsoft Excel. All discrepancies during the final round of coding were first discussed between the coder and checker, and if still unresolved, discussions took place within the entire study group until consensus was reached. The most discrepant moderators during this final round of coding were 'unspecified social support' (BCT; 9 resolved discrepancies); 'information about health consequences', 'information about social and environmental consequences', and 'body changes' (BCTs; 6 resolved discrepancies each); and 'feedback on behavior' (BCT; 5 resolved discrepancies).

After coding, outcome data were extracted and input to Comprehensive MetaAnalysis software v3 (CMA; Borenstein, Hedges, Higgins \& Rothstein, 2014) by one researcher (KK or MB) and verified by another (MB or KK). Outcome data included all measures of intention, stage of change, autonomous motivation and physical activity for each study group at baseline and first post-treatment measurement point. Corresponding authors were contacted via phone or email to try to obtain any missing data or additional information needed to calculate effect sizes.

\section{Statistical Procedures}

All analyses were either prespecified in the registration of this review or were suggested during the peer review process. 
Meta-analyses were conducted within CMA, and effect sizes were computed by entering means and standard deviations at baseline and post-treatment, standardized by the pooled standard deviation and corrected for pre-post correlations within groups (Morris \& De Shon, 2002). For studies where this information was not available, alternative comparable methods were used (e.g. F-ratio and p-value, mean change scores, previously computed effect sizes such as Cohen's d), or the pre-post correlation was imputed using the mean of all other pre-post correlations available from interventions reporting on that outcome (Morris \& De Shon, 2002). To calculate the effect sizes for stage of change outcomes, the action and maintenance stages were collapsed into one post-intentional stage, and within-groups effect sizes were calculated by comparing the distributions of individuals in each stage at baseline and post-treatment. This method has been described in a book by Lipsey and Wilson (2001), and calculations of this type were undertaken with a freely-available online calculator created by the authors of the book (Wilson, 2001). Intention-to-treat data were used when available. For studies with only complete case data, effect sizes were calculated based on the number of cases for which post-treatment data were available (i.e. not the full enrolled sample).

Cumulative effect size data were combined using random effects meta-analyses in CMA. Cohen's $d$ values with corresponding 95\% confidence intervals and two-sided p-values were used as the primary measure of cumulative effect size, and indications of heterogeneity were examined with $\mathrm{I}$-squared statistics. Outlying data points (studies with effect sizes further than three standard deviations from the mean cumulative effect size) were Winsorized and replaced with the next most extreme allowable value (Harkin et al, 2016). Publication bias was examined with funnel plots and trim and fill methods (Duval \& Tweedie, 2000).

Comparative subgroup analyses were used to identify BCTs and other moderators associated with changes in motivational outcomes. For each moderator which was both present and absent in at least three arms reporting on a specific outcome, a subgroup analysis 
within CMA compared the cumulative effect size of interventions which included the moderator to the cumulative effect size of interventions which did not include it. Effect sizes for these comparisons were computed using the methods of Borenstein, Hedges, Higgins and Rothstein (2009). Additional subgroup analyses and meta-regressions within CMA were used to examine the associations between effect sizes and factors related to study design and population including age, disease status, overweight status, baseline sedentary behavior status, recruitment methods, intervention setting, mode of delivery (digital vs other; group vs individual; mobile vs other; face-to-face vs self-guided), total number of BCTs used, contact time, contact sessions, time in weeks between baseline and post-treatment, and stated theoretical basis.

Finally, meta-regression analyses and moving constant analyses (Johnson \& HuedoMedina, 2011) examined the extent to which the effects of interventions on intention, stage of change and autonomous motivation predicted the effects of interventions on measures of physical activity.

\section{Results}

\section{Identification of Studies}

The PRISMA flow diagram in Figure 1 provides details on the search and study selection procedures, which identified 89 studies that reported baseline to post-treatment changes in either intention to be physically active, autonomous motivation or stage of change.

\section{Descriptive Study Characteristics}

Of the 89 included studies, 78 reported data from multiple groups and 11 reported data from single study arms only. These studies included 200 study arms overall, comprising 19,212 participants. Outcome data on intention, stage of change and autonomous motivation were reported in 77, 96 and 34 study arms respectively. Supplementary File 1 provides details 
of all included study arms, including settings, treatment descriptions, and demographic information of the study samples. All supplementary files are available at https://osf.io/2far3/.

\section{Behavior Change Techniques}

In coding the included studies for their use of BCTs, three additional BCTs were identified that were not sufficiently covered by the v1 taxonomy (Michie et al., 2013).

Definitions for each of these were discussed and standardized within the research team and added to the taxonomy to inform subsequent coding. The newly identified BCTs were: 17.1) 'provision of pedometer or other wearable device', which was defined to include measurement devices that could act as a cue to behavior, such as pedometers, heart rate monitors and accelerometers, but which were not formally part of an intervention strategy; 17.2) 'motivational interviewing', for which the definition provided in a previous BCT taxonomy was used (Michie et al., 2011); and 17.3) instructing individuals on aspects of the behavior to be carried out', which was coded in instances where the interventionist specified the modality, intensity, time or location of the behavior to be performed (as opposed to specifying the quantity or frequency of the behavior, which would have then been coded as behavioral goal setting). These newly identified BCTs were identified in 28, 17 and 65 study arms, respectively.

Across the 200 coded arms of the included studies, 69 of the 96 possible BCTs were identified as present in at least one study arm, and the most commonly occurring BCTs were behavioral goal setting $(\mathrm{k}=108)$, providing information on health consequences $(\mathrm{k}=88)$, problem solving $(\mathrm{k}=71)$, action planning $(\mathrm{k}=68)$, instructing on aspects of the behavior to be carried out $(\mathrm{k}=65)$, and behavioral self-monitoring $(\mathrm{k}=63)$. The most intensive study arm included 23 BCTs delivered across a 12-week exercise counselling program (Kim et al, 2004), and 42 arms (mainly no-treatment or waiting list control arms) did not include any 
codable BCT content. Full information on the BCTs included in each intervention arm is available in Supplementary File 2, and additional intervention-level moderators are included in Supplementary File 1.

\section{Cumulative Effect Sizes}

To examine the effects of interventions upon motivational constructs when compared to control groups, cumulative effect sizes were calculated across RCT studies. The largest effects of interventions were found in studies which reported on autonomous motivation $(d=$ $0.32 ; 95 \%$ CI $\left.[0.13,0.50] ; p=.001 ; k=20 ; \mathrm{I}^{2}=77.62\right)$, with smaller cumulative effect sizes evident for intention $\left(d=0.17 ; 95 \%\right.$ CI $\left.[0.08,0.26] ; p<.001 ; k=41 ; \mathrm{I}^{2}=53.82\right)$ and stage of change $\left(d=0.19 ; 95 \%\right.$ CI $\left.[0.10,0.28] ; p<.001 ; k=48 ; \mathrm{I}^{2}=60.37\right)$. Cumulative effects on stage of change revealed some publication bias, and imputing unpublished studies using trim and fill procedures (Duval \& Tweedie, 2000) resulted in a smaller cumulative effect size of $d$ $=0.12(95 \%$ CI $[0.02,0.21])$. Cumulative effects were also calculated for individual study arms, when not compared to control groups. Forest plots for randomized controlled trials and individual study arms, as well as funnel plots for publication bias are presented in Supplementary File 3. These cumulative effects indicated considerable heterogeneity, which we subsequently sought to examine with moderator analyses.

\section{Moderator Analyses}

Behavior change techniques. Moderator analyses revealed several BCTs associated with changes in motivational constructs. Six BCTs were associated with beneficial changes in intention and 14 BCTs with beneficial changes in stage of change, while one BCT (demonstration of behavior) was associated with beneficial changes in autonomous motivation. The presence of behavioral goal setting, self-monitoring of behavior, and behavioral practice or rehearsal were each independently associated with beneficial changes in two motivational outcomes. Furthermore, four BCTs were found to be independently 
associated with adverse changes in stage of change, with effect sizes ranging from $d=-0.47$ to $d=-0.21$. Table 1 provides effect sizes and confidence intervals for comparative subgroup analyses of BCTs for which at least one significant moderation effect occurred. Full data from all conducted comparative subgroup analyses are available in Supplementary File 4.

Modes of delivery. In examining modes of delivery as potential moderators of effect sizes, interventions which included face-to-face delivered components produced significantly larger effect sizes $(p<.05)$ than interventions which did not include face-to-face delivered components on all three motivational constructs under study. Interventions which included group-delivered components produced significantly larger effects on intention and stage of change than interventions without any group-delivered components. Furthermore, interventions which included telephone follow-ups, took place in gyms or fitness centers or were delivered by gym workers had larger effects on stage of change and autonomous motivation than interventions delivered in other settings. Interventions which included contacts via postal mail were significantly associated with unfavorable changes in intention and autonomous motivation. Several other mode of delivery aspects were significantly associated with one single outcome under study. See Table 2.

Participant characteristics. Characteristics of the study samples (including whether the sample presented with a chronic illness, included only sedentary individuals at baseline or included only overweight individuals) were also examined as moderators of effect size. Interventions delivered exclusively to sedentary individuals produced greater effects on stage of change than interventions which did not exclude active individuals $(d=0.48)$.

Interventions delivered exclusively to overweight individuals produced greater effects on stage of change and autonomous motivation than interventions which did not exclude individuals of normal weight. No other participant characteristics moderated effect size for any other outcomes (Table 2). 


\section{Meta-Regression Analyses}

\section{Relationships between continuous moderators and changes in motivational}

variables. A greater number of included BCTs was associated with greater intervention effects on intention $\left(b=0.02, k=77, p=.002, \mathrm{R}^{2}=.08\right)$ and stage of change $(b=0.03, k=$ $\left.96, p<.001, \mathrm{R}^{2}=.07\right)$, but not autonomous motivation $\left(b=0.01, k=34, p=.144, \mathrm{R}^{2}=.01\right)$. Effect sizes for changes in intention to be physically active were not significantly associated with any other continuous moderators under study (sample gender, BMI, number of treatment contacts, contact hours). Effect sizes for stage of change and autonomous motivation were however both significantly associated with an increased BMI in the sample (for SoC: $b=$ $0.06, k=48, p<.001, \mathrm{R}^{2}=.34$; for autonomous motivation: $\hat{b}=0.04, k=26, p=.002, \mathrm{R}^{2}=$ .36). Effect sizes for stage of change were furthermore significantly associated with a higher percentage of female participants $\left(b=.01, k=95, p<.001, \mathrm{R}^{2}=.00\right)$, a greater number of treatment contacts $\left(b=0.01, k=67, p<.001, \mathrm{R}^{2}=.03\right)$, and a greater number of intervention contact hours $\left(b=0.01, k=50, p=.012, \mathrm{R}^{2}=.00\right)$. There were no significant relationships between length of follow-up period (weeks from baseline) and effect size for any of the variables under study. Outputs of all meta-regression analyses are presented in Supplementary File 5.

\section{Relationships between changes in motivation variables and changes in physical}

activity. Effect sizes for changes in physical activity (both objective and subjective measures) were moderately associated with effect sizes for changes in intention $(b=0.55, k=$ $\left.54, p<.001, \mathrm{R}^{2}=.20\right)$ and less strongly associated with effect sizes for stage of change $(b=$ $\left.0.31, k=57, p=.001, \mathrm{R}^{2}=.08\right)$, but not significantly associated with effect sizes for changes in autonomous motivation $\left(b=0.31, k=22, p=.251, \mathrm{R}^{2}=.07\right)$.

Moving constant analyses revealed that the confidence interval for intervention effects on physical activity is not likely to include zero when interventions have effects on intention 
or autonomous motivation at a magnitude of $d>-0.20$, or effects on stage of change at a magnitude of $d>0.05$. Confidence intervals for the expected effects on physical activity at each level of effect size for motivational outcomes are presented graphically in Supplementary File 6.

\section{Discussion}

The present study sought to identify characteristics of physical activity interventions associated with changes in intention, stage of change and autonomous motivation - the seminal motivational constructs proposed by several prominent behavioral theories. Of all potential moderators examined, only face-to-face intervention delivery was associated with beneficial changes in all three motivational outcomes under study. In total, 18 BCTs, ten modes of delivery and four other study characteristics moderated the effects of interventions on at least one motivational outcome, and these significant moderators seemed to cluster in several ways.

\section{Moderators of Changes in Motivational Outcomes}

Behavior change techniques and modes of delivery. Interventions including BCTs derived from control theory (i.e. behavioral goal setting, action planning, self-monitoring of behavior, feedback on behavior, and problem solving) (Carver \& Scheier, 1982) were associated with greater changes in intention and stage of change than other interventions. Inclusion of any control theory BCT was associated with progression in stage of change, with effect sizes in the 0.20-0.30 range; and inclusion of either 'behavioral goal setting' or 'selfmonitoring of behavior' was associated with favorable changes in intention, with smaller effects of 0.12 and 0.24 respectively. The association between behavioral goal setting and effect sizes for intention is in line theoretical assumptions and reflects a direct route between goals and intention formation (Maes \& Karoly, 2005). Despite its similarities to self- 
liberation from the transtheoretical model, we were unable to examine the impact of the BCT 'commitment' on stage of change, as too few studies reported utilizing this technique.

Applying the same method as a previous meta-analysis on physical activity and healthy eating interventions (Michie et al., 2009), our analyses showed that interventions including self-monitoring of behavior plus any other control theory BCT produced greater changes in intention and stage of change than interventions which did not include this set of BCTs, with effect sizes around 0.25. Control theory BCTs were also among those most commonly identified as present in the included interventions. Within previous meta-analyses of physical activity interventions, interventions including control theory BCTs have led to greater changes in behavior than those which did not (Avery et al., 2012; Dombrowski et al., 2012; Knittle, Maes \& De Gucht, 2010; Michie et al., 2009). Although the effect sizes for the individual impact of these control theory techniques are small, these techniques seem integral to changing motivation, especially considering their previously-identified effects on behavior.

Interventions including exercise classes typically included the following BCTs: 'instruction on how to perform the behavior', 'behavioral practice or rehearsal', and 'demonstration of behavior' (Michie et al., 2013). These three BCTs each produced effect sizes of around 0.40 for stage of change; 'behavioral practice or rehearsal' led to small effects on intention; and 'demonstration of behavior', with an effect size of 0.19 , was the lone BCT significantly associated with increases in autonomous motivation. In addition, delivery in gym settings produced large effects of 0.74 on stage of change, while interventions delivered in group settings and via face-to-face interactions were each associated with small changes in all motivational outcomes, apart from group delivery and autonomous motivation, where there was no association. These BCTs and modes of delivery seem to form a cluster related to exercise class attendance, and may alter motivational outcomes via the hands-on experiences that help to make a new behavior achievable, familiar, and (ideally) enjoyable, as well as 
connecting the individual to other people socially. Offering individuals opportunities to try the target behavior (e.g. behavioral practice) and prompting preparations for behavior during the intervention, regardless of an individual's motivational status (Sutton, 2008), may be a good means for increasing motivation. Consistent with theories, practicing skills and receiving meaningful first-hand feedback in a social setting may furthermore influence individuals' perceptions of personal capacities and perceived constraints regarding the target behavior, increasing perceived behavioral control and normative beliefs from the theory of planned behavior (Hagger \& Chatzisarantis, 2009) and fulfilling needs for competence and relatedness from self-determination theory (Ryan \& Patrick, 2009).

Although face-to-face and group-delivered interventions had significant positive effects on motivational outcomes, BCTs related to social support and social influences were not significantly associated with any motivational outcomes. Furthermore, the BCTs 'practical social support' (e.g., prompting an individual to find an exercise buddy or source of social support) and 'restructuring the social environment' (e.g., workplace weight loss or physical activity competitions), as well as intervention delivery by a peer facilitator or a physiotherapist, were associated with negative changes in stage of change. While it should be noted that these negative findings come from imbalanced comparisons, as each moderator was present in five or fewer studies, this seeming contradiction indicates that a mix of opportunities for both upward and downward comparisons may be ideal for increasing motivation (Collins, 1996), and indicates the need for closer examinations of how the quality and content of social support and social interactions impact on intervention effectiveness. As an example, experiencing coercion or external pressure from others is likely to lead to negative changes in motivation and behavior (Deci \& Ryan, 2000), but being surrounded by others who face similar challenges is likely to have a positive impact. To shed light on the impact of social interactions, studies should make efforts to thoroughly describe delivered 
interventions and make use of new taxonomies which can capture qualitative differences in social interactions (Hardcastle, Fortier, Blake \& Hagger, 2017).

Within this study, few intervention components or modes of delivery were associated with changes in autonomous motivation. Techniques such as motivational interviewing and various forms of social support, which have previously been theorized to foster autonomous motivation (Markland \& Vansteenkiste, 2007; Markland et al., 2005) showed no significant effects or could not be examined due to lack of studies. This lack of effects could potentially be attributable to limited statistical power, but may also indicate that the mechanisms of change for autonomous motivation operate through channels other than the BCTs present in the v1 taxonomy (Michie et al., 2013). While still limited by incomplete intervention descriptions, the use of newly-developed taxonomies which list techniques derived from motivational interviewing (Hardcastle et al., 2017) and techniques specifically identified to satisfy the basic needs proposed within self-determination theory (Teix eira \& Hagger, 2016) could potentially identify additional intervention factors which moderate effects on autonomous motivation. It should also be noted that the construct autonomous motivation includes factors related to enjoyment (i.e. intrinsic motives), as well as habits and congruence with personal values (i.e. integrated and identified regulations, respectively). As such, the BCTs examined here may have altered one form of autonomous motivation but not the entire autonomous motivation construct. It was not possible to examine this however, as many studies utilized autonomous motivation measures which made no distinctions between intrinsic, integrated and identified regulatory styles. Future intervention studies should therefore utilize measures which can distinguish between them.

Meta-regression analyses revealed a positive association between the number of BCTs an intervention included and the magnitude of its effects on intention and stage of change. This relationship did not hold however for changes in autonomous motivation. In line with 
previous meta-analyses demonstrating a link between a greater number of included BCTs and larger effect sizes on physical activity (Hynynen et al., 2016; Webb, Joseph, Yardley \& Michie, 2010), our analyses suggest that interventions which involve more BCTs lead to greater changes in motivational for physical activity as well. However, more is not necessarily better, and choices of which BCTs to include within an intervention should be guided by theory-driven mechanisms of action (Michie et al, 2016), as well as the time and resources available for intervention delivery.

Theory-based interventions. Interventions explicitly targeting behavioral determinants from the theory of planned behavior (including reasoned action approach and health action process approach models) or social cognitive theory produced greater effect sizes on intention and stage of change than studies which did not target these constructs. This finding extends those of previous meta-analyses, which had found that internet-based interventions based on the theory of planned behavior had greater effects than other interventions (Webb et al., 2010), and that interventions explicitly based on social cognitive theory significantly increase physical activity among cancer survivors (Stacey, James, Chapman, Courneya \& Lubans, 2015). Given the important theoretical position of selfefficacy cognitions within both social cognitive theory and the theory of planned behavior, and the well-defined direct links between self-efficacy and behavior in multiple domains, our results confirm the importance of fostering cognitions related to personal control over behavior in influencing both motivation and physical activity behavior.

Sample characteristics. Studies which included only overweight or obese individuals yielded larger effect sizes on stage of change and autonomous motivation than studies which did not have weight as an inclusion criterion. Higher BMI was also associated with greater changes in stage of change and autonomous motivation. These findings could be explained by the inverse relationships between BMI and autonomous motivation and stage of change for 
physical activity reported previously (Markland \& Ingledew, 2007; Wee, Davis \& Phillips, 2005), which could have resulted in floor effects (i.e., more scope for improving). Our finding that studies which only included sedentary individuals had larger effects on stage of change than studies which made no such restrictions could potentially be explained by floor effects as well. To identify which intervention methods work best for whom, future studies should examine interactions between characteristics of individuals and BCT content, ideally on a per-participant level instead of trial-level.

\section{Cumulative Effect Sizes}

While not the primary aim of this meta-analysis, this study investigated the cumulative effects of physical activity interventions on intention, stage of change and autonomous motivation. Relative to control groups, active intervention arms produced small and significant cumulative effects on these motivational constructs, which is consistent with findings from a meta-analysis which investigated the effects of interventions on self-efficacy (French, Olander, Chisholm \& Mc Sharry, 2014). The small effect sizes found here differ from previous meta-analyses which found larger cumulative effect sizes of $d=0.45$ and $d=$ 0.66 for changes in intention (Steinmetz et al., 2016; Rhodes \& Dickau, 2012; Webb \& Sheeran, 2006). As this meta-analysis included nearly 15 more studies than the next most recent meta-analysis on the topic (Steinmetz et al., 2016), the smaller cumulative effect of $d$ $=0.17$ may better estimate the true effects of interventions on physical activity intentions.

\section{Associations between Changes in Motivation Outcomes and Behavior}

Of the three motivational constructs under study here, changes in intention

demonstrated the strongest relationship with contemporaneous changes in physical activity $(b$ $=0.55)$, and at a level comparable to the correlations of $r=.51$ and $r=.57$ found in previous meta-analyses on the topic (Rhodes \& Dickau, 2012; Webb \& Sheeran, 2006). Despite the strength of this relationship, considerable evidence for the intention-behavior gap remains 
(Sheeran \& Webb, 2016). Automatic and non-intentional routes to (increasing) physical activity merit considerable attention when developing predictive models and developing future interventions and theories (Hagger \& Chatzisarantis, 2014), although these were not part of this review's focus on primarily goal-directed behavior.

Changes in stage of change were also associated with changes in physical activity. This is consistent with the findings of Armitage and Arden (2002), who examined the ability of theory of planned behavior variables to predict stage transitions within the transtheoretical model, and could be explained by their conclusion that stage of change may function as a proxy measure of behavior, as opposed to capturing distinct social cognitions. In calculating effect sizes for stage of change outcomes in this study, we attempted to mitigate the effects of the entanglement of behavioral, intentional and cognitive factors in stage of change assessment items by collapsing the action and maintenance stages. However, it is not fully possible to disentangle these variables, and a chance remains that the strength of relationship found is due in part to this measurement non-specificity.

Despite the interventions included here producing larger cumulative effect sizes on autonomous motivation than on either intention or stage of change, no significant relationship existed between changes in autonomous motivation and changes in physical activity behavior. This might be attributable to a lack of power to detect a significant relationship in this analysis however, as the regression coefficient for autonomous motivation $(b=0.31)$ was nearly identical to that between stage of change and physical activity, where a significant relationship was found. Despite this possibility, the main finding here is in line with previous research indicating that self-determination theory constructs better explain physical activity maintenance than they do physical activity initiation (Wasserkampf et al., 2014), but somewhat conflicts with previous meta-analyses that had demonstrated links between autonomous motivation, intention and physical activity behavior (Hagger \& Chatzisarantis, 
2009; Ng et al., 2012). Previous meta-analyses had not investigated relationships between changes in these variables however, and the lack of a relationship between changes in autonomous motivation and physical activity could indicate that interventions failed to assist individuals in transferring new behavioral routines from intervention contexts into daily life. For example, interventions which included consistent attendance to exercise classes or coaching may have resulted in changes in autonomous motivation (i.e. more enjoyment of behavior), but not necessarily in behavioral enactment after the conclusion of the exercise classes or coaching. Interventions which include significant amounts of behavioral practice should be combined with self-regulatory strategies to keep activities going in the absence of formal instruction and to help translate autonomous motivation into sustained action (Nurmi et al., 2016).

\section{Motivation and the First Steps toward Behavior Change}

While the current study examined how intervention components relate to increases in motivation once an individual has taken part in a physical activity intervention, it does not shed light on the best methods for getting people interested in participating in physical activity interventions in the first place. One might be interested in an intervention aimed at weight reduction, for example, but not motivated to exercise daily. Or conversely: One might be motivated to avoid cardiovascular disease, but still not be interested in taking part in a physical activity intervention (Crutzen \& Ruiter, 2015). In other words, intervention uptake is itself a behavior which is influenced by specific determinants, but this has received limited attention in the currently-dominant efficacy-based paradigm (Kohl, Crutzen, \& De Vries, 2013). As intervention uptake is not necessarily dependent on the content of an intervention itself, meta-interventions may help to stimulate interest in intervention participation (Albarracín, Durantini, Earl, Gunnoe, \& Leeper, 2008). Previous experimental studies on meta-interventions have focused on using various Google AdWords (Crutzen, Ruiter, \& De 
Vries, 2014) and gender-tailored brochures (McCulloch, Albarracín, \& Durantini, 2008). To optimize such meta-interventions, however, it is crucial to gain more insight into determinants of intervention uptake and to link the content of these meta-interventions to these determinants. The BCTs identified here as associated with changes in motivational constructs could serve as an initial set of testable intervention components to increase both uptake of physical activity interventions and deliberative motivational constructs toward physical activity.

\section{Study Strengths and Limitations}

The current study involved robust and replicable search, screening and coding procedures, and followed recommendations put forth in the Iterative Protocol for Evidence Base Accumulation (Peters et al., 2015) and PRISMA (Moher, Liberati, Tetzlaff \& Altmann, 2009) statements. BCT content and modes of delivery were coded using consensus procedures, and the resolved discrepancies in coding may indicate the need for refinement of BCT definitions for information provision and social support in the v1 taxonomy (Michie et al., 2013). Coding was done separately for intervention and control groups, as the BCTs and modes of delivery offered by active and control interventions can overlap considerably (De Bruin et al., 2010). Without knowing whether a BCT was being tested in the first place (i.e., delivered exclusively in the intervention group), it impossible to draw conclusions about which BCTs work and which do not (Peters et al., 2015). The coding method employed here, coupled with moderator analyses based on within-group (as opposed to between-groups) effect sizes (Morris \& De Shon, 2002), allows for a more straightforward examination of how active intervention content affects outcomes. As this study investigated moderators of intervention effectiveness for multiple theoretical conceptualizations of motivation (i.e., intention, stage of change, and autonomous motivation), the findings will be of interest to 
researchers from various theoretical backgrounds. Future research is needed to examine the direct associations between intention, stage of change and autonomous motivation and the extent to which the moderators identified here increase motivation in other domains of health behavior.

While the large sample sizes in this study offered considerable power in detecting moderator effects, causal inferences should not be drawn based on the identified significant associations. All findings should instead serve as a tool from which hypotheses for experimental studies can be generated and new evidence-based interventions can be developed (Peters et al., 2015).

Several other cautionary notes should guide interpretations of the results: Moderator analyses conducted for BCTs and other moderators present in only a small number of interventions (e.g., mental rehearsal of successful behavioral performance, which was present in only six interventions reporting on intention for physical activity) may have been imbalanced, and should be interpreted with caution. Publication bias, the exclusion of 96 studies for which appropriate or additional data could not be obtained from study authors, and the possibility of coincidental co-occurrence of effective BCTs in the 'absent' side of comparisons may also have affected results (Peters et al., 2015). Furthermore, post hoc analyses revealed that higher study dropout rates were significantly associated with smaller effect sizes for intention, which may have influenced results. This finding could indicate that a failure to feel more motivated causes some individuals to drop out of interventions, and points at additional variables (e.g. self-control) which may be necessary precursors for individuals to engage with interventions (Hagger, Wood, Stiff \& Chatzisarantis, 2010). Finally, this study assessed the effects of moderators one at a time, so we cannot speculate on how patterns of co-occurrence and interactions between BCTs and modes of delivery might have influenced the results. Further analyses involving classification and regression trees 
(Dusseldorp, Van Genugten, Van Buuren, Verheijden, \& Van Empelen, 2004) could potentially be used to model how organic patterns of co-occurrence impact upon motivational outcomes in future studies.

The BCT coding procedures undertaken in this study relied on the text present in intervention descriptions from published articles, supplementary materials and any secondary references provided by the authors. While this method is often used in meta-analyses and captures intervention content reasonably well (Presseau et al., 2015), some BCT content may have been missed due to incomplete intervention descriptions. Other limitations of this method exist as well: First, it does not make it clear whether BCTs were applied correctly during an intervention. As the effectiveness of an intervention component depends on whether its parameters for use are satisfied (e.g., although modelling of behavior can be an effective BCT, a modelling case where a celebrity begins exercising instantly and effortlessly is unlikely to contribute to behavior change; [Peters, De Bruin, \& Crutzen, 2015]). Second, this coding method does not provide any information on whether the coded BCTs were delivered as intended and uniformly to all intervention participants (i.e., intervention fidelity; [Knittle, 2015]). While information on fidelity is rarely reported (especially at the BCT level), it is a major issue affecting inferences that can be made (De Bruin, Crutzen, \& Peters, 2015). Finally, even with high fidelity of delivery, enactment of BCTs by participants may be suboptimal (e.g., participants might not complete self-monitoring records or action plans), which can also affect outcomes (Hankonen et al., 2015; Knittle et al., 2016). Such aspects of actual intervention content could not be accounted for in the present study. Hence, we would like to echo previous calls to improve reporting quality of intervention development and evaluation research (Albrecht, Archibald, Arseneau, \& Scott, 2013; Knittle, 2015).

\section{Conclusion}


This is, to our knowledge, the first study to identify BCTs and intervention features associated with changes in motivation for physical activity, as conceptualized in several influential behavioral theories. The results indicate that self-monitoring, goal setting, and other self-regulatory BCTs play a significant role in changing intention and stage of change, as they do with physical activity behavior itself. Additionally, interventions delivered face-toface and which contained components frequently delivered as part of exercise classes resulted in greater changes in intention, stage of change and autonomous motivation. While the added effect of including each significant moderator was small, the results can be used in designing interventions and experimental studies to increase motivation and encourage uptake of selfregulatory interventions targeting physical activity behavior change. Future research should investigate whether similar patterns also hold when examining changes in motivation in relation to other health behaviors. 


\section{References}

References included in the meta-analyses are presented in the Appendix.

Adams, J., \& White, M. (2005). Why don't stage-based activity promotion interventions work?. Health Education Research, 20(2), 237-243, https://doi.org/10.1093/her/cyg105.

Ajzen, I. (1991). The theory of planned behavior. Organizational Behavior and Human Decision Processes, 50, 179-211, https://doi.org/10.1016/0749-5978(91)90020-T.

Ajzen, I. (2002). Perceived behavioral control, self- efficacy, locus of control, and the theory of planned behavior. Journal of Applied Social Psychology, 32, 665-683, doi:10.1111/j.15591816.2002.tb00236.x.

Albarracín, D., Durantini, M. R., Earl, A., Gunnoe, J. B., \& Leeper, J. (2008). Beyond the most willing audiences: a meta-intervention to increase exposure to HIV-prevention programs by vulnerable populations. Health Psychology, 27, 638-644, doi:10.1037/0278-6133.27.5.638.

Albrecht, L., Archibald, M., Arseneau, D., \& Scott, S. D. (2013). Development of a checklist to assess the quality of reporting of knowledge translation interventions using the Workgroup for Intervention Development and Evaluation Research (WIDER) recommendations.

Implementation Science, 8, 52, https://doi.org/10.1186/1748-5908-8-52.

Armitage, C. J. (2009). Is there utility in the transtheoretical model?. British Journal of Health Psychology, 14, 195-210, doi:10.1348/135910708X368991.

Avery, L., Flynn, D., Van Wersch, A., Sniehotta, F. F., \& Trenell, M. I. (2012). Changing Physical Activity Behavior in Type 2 Diabetes A systematic review and meta-analysis of behavioral interventions. Diabetes Care, 35, 2681-2689, doi:10.2337/dc11-2452.

Armitage, C. J., \& Arden, M. A. (2002). Exploring discontinuity patterns in the transtheoretical model: An application of the theory of planned behaviour. British Journal of Health Psychology, 7, 89-103, doi:10.1348/135910702169385.

Bandura, A. (1977). Self-efficacy: toward a unifying theory of behavioral change. Psychological Review, 84, 191-215, https://doi.org/10.1016/0146-6402(78)90002-4. 
Borenstein, M., Hedges, L., Higgins, J., \& Rothstein, H. (2014). Comprehensive Meta-Analysis (Version 3.3.070) [Software]. Available from www.meta-analysis.com

Borenstein, M., Hedges, L., Higgins, J., \& Rothstein, H. (2009). Introduction to meta-analysis. Hoboken, NJ: Wiley.

Carver, C. S., \& Scheier, M. F. (1982). Control theory: a useful conceptual framework for personalitysocial, clinical and health psychology. Psychological Bulletin, 92, 111-135, doi:10.1037/00332909.92.1.111.

Caspersen, C. J., Powell, K. E., \& Christenson, G. M. (1985). Physical activity, exercise, and physical fitness: definitions and distinctions for health-related research. Public Health Reports, 100(2), 126-131. PMID:3920711

Collins, R. L. (1996). For better or worse: The impact of upward social comparison on selfevaluations. Psychological Bulletin, 119, 51-69, http:/dx.doi.org/10.1037/0033-2909.119.1.51.

Colman, A. M. (Ed.). (2014). The Oxford Dictionary of Psychology (3rd ed.). Oxford, UK: Oxford University Press. eISBN:9780191726828

Crutzen, R., \& Ruiter, R. A. C. (2015). Interest in behaviour change interventions: a conceptual model. The European Health Psychologist, 17, 6-11, http://www.ehps.net/ehp/index.php/contents/article/view/759/pdf_35.

Crutzen, R., Ruiter, R. A. C., \& De Vries, N. K. (2014). Can interest and enjoyment help to increase use of Internet-delivered interventions? Psychology \& Health, 29, 1227-1244, doi:10.1080/08870446.2014.921300.

De Bruin, M., Crutzen, R., \& Peters, G.-J. Y. (2015). Everything should be as simple as possible, but this will still be complex: a reply to various commentaries on IPEBA. Health Psychology Review, 9, 38-41, http://dx.doi.org/10.1080/17437199.2014.981833.

De Bruin, M., Viechtbauer, W., Schaalma, H. P., Kok, G., Abraham, C., \& Hospers, H. J. (2010). Standard care impact of effects of highly active antiretroviral therapy adherence interventions: A meta-analysis of randomized controlled trials. Archives of Internal Medicine, 170, 240-250, doi:10.1001/archinternmed.2009.536. 
Deci, E. L., \& Ryan, R. M. (2000). The" what" and" why" of goal pursuits: Human needs and the selfdetermination of behavior. Psychological Inquiry, 11, 227-268, http://dx.doi.org/10.1207/S15327965PLI1104_01.

Ding, D., Lawson, K. D., Kolbe-Alexander, T. L., Finkelstein, E. A., Katzmarzyk, P. T., van Mechelen, W., ... \& Lancet Physical Activity Series 2 Executive Committee. (2016). The economic burden of physical inactivity: a global analysis of major non-communicable diseases. The Lancet, 388, 1311-1324, doi:10.1016/S0140-6736(16)30383-X.

Dombrowski, S. U., Sniehotta, F. F., Avenell, A., Johnston, M., MacLennan, G., \& Araújo-Soares, V. (2012). Identifying active ingredients in complex behavioural interventions for obese adults with obesity-related co-morbidities or additional risk factors for co-morbidities: a systematic review. Health Psychology Review, 6, 7-32, http://dx.doi.org/10.1080/17437199.2010.513298.

Dusseldorp, E., Van Genugten, L., Van Buuren, S., Verheijden, M. W., \& Van Empelen, P. (2004). Combinations of techniques that effectively change health behavior: evidence from MetaCART analysis. Health Psychology, 33, 1530-1540, doi:10.1037/hea0000018.

Duval, S., \& Tweedie, R. (2000). Trim and fill: a simple funnel- plot-based method of testing and adjusting for publication bias in meta- analysis. Biometrics, 56(2), 455-463, DOI:10.1111/j.0006-341X.2000.00455.x.

Fishbein, M., \& Ajzen, I. (2011). Predicting and changing behavior: The reasoned action approach. New York, NY: Taylor \& Francis.

French, D. P., Olander, E. K., Chisholm, A., \& Mc Sharry, J. (2014). Which behaviour change techniques are most effective at increasing older adults' self-efficacy and physical activity behaviour? A systematic review. Annals of Behavioral Medicine, 48, 225-234, doi: $10.1007 / \mathrm{s} 12160-014-9593-\mathrm{z}$.

Gollwitzer, P. M. (1990). Action phases and mind-sets. In R. M. Sorrentino \& E. T. Higgins (Eds.), Handbook of motivation and cognition: Foundations of social behavior (pp. 53-92). New York, NY: Guilford Press. 
Hagger, M., Chatzisarantis, N. L., Hein, V., Soós, I., Karsai, I., Lintunen, T., \& Leemans, S. (2009). Teacher, peer and parent autonomy support in physical education and leisure-time physical activity: A trans-contextual model of motivation in four nations. Psychology and Health, 24(6), 689-711, https://doi.org/10.1080/08870440801956192.

Hagger, M. S., \& Chatzisarantis, N. L. (2009). Integrating the theory of planned behaviour and selfdetermination theory in health behaviour: A meta- analysis. British Journal of Health Psychology, 14, 275-302, doi:10.1348/135910708X373959.

Hagger, M. S., \& Chatzisarantis, N. L. (2014). An integrated behavior change model for physical activity. Exercise and Sport Sciences Reviews, 42(2), 62-69, doi: 10.1249/JES.0000000000000008.

Hagger, M. S., Wood, C. W., Stiff, C., \& Chatzisarantis, N. L. (2010). Self-regulation and self-control in exercise: The strength-energy model. International Review of Sport and Exercise Psychology, 3(1), 62-86, https://doi.org/10.1080/17509840903322815.

Hankonen, N., Sutton, S., Prevost, A. T., Simmons, R. K., Griffin, S. J., Kinmonth, A. L., \& Hardeman, W. (2015). Which behavior change techniques are associated with changes in physical activity, diet and body mass index in people with recently diagnosed diabetes?. Annals of Behavioral Medicine, 49, 7-17, doi:10.1007/s12160-014-9624-9.

Hankonen, N., Heino, M. T., Kujala, E., Hynynen, S. T., Absetz, P., Araújo-Soares, V., ... \& Haukkala, A. (2017). What explains the socioeconomic status gap in activity? Educational differences in determinants of physical activity and screentime. BMC Public Health, 17(1), 144, https://doi.org/10.1186/s12889-016-3880-5.

Hardcastle, S. J., Fortier, M., Blake, N., \& Hagger, M. S. (2017). Identifying content-based and relational techniques to change behaviour in motivational interviewing. Health Psychology Review, 11, 1-16, http://dx.doi.org/10.1080/17437199.2016.1190659.

Harkin, B., Webb, T. L., Chang, B. P. I., Prestwich, A., Conner, M., Kellar, I., ... \& Sheeran, P. (2016). Does Monitoring Goal Progress Promote Goal Attainment? A Meta-Analysis of the Experimental Evidence. Psychological Bulletin, 142, 198-229, doi:10.1037/bul0000025. 
Harrison, R. A., Roberts, C., \& Elton, P. J. (2004). Does primary care referral to an exercise programme increase physical activity one year later? A randomized controlled trial. Journal of Public Health, 27(1), 25-32, https://doi.org/10.1093/pubmed/fdh197.

Henrich, J. F., Knittle, K., De Gucht, V., Warren, S., Dombrowski, S. U., \& Maes, S. (2015). Identifying effective techniques within psychological treatments for irritable bowel syndrome: A meta-analysis. Journal of Psychosomatic Research, 78, 205-222, doi:10.1016/j.jpsychores.2014.12.009.

Hobbs, N., Godfrey, A., Lara, J., Errington, L., Meyer, T. D., Rochester, L., ... \& Sniehotta, F. F. (2013). Are behavioral interventions effective in increasing physical activity at 12 to 36 months in adults aged 55 to 70 years? a systematic review and meta-analysis. BMCMedicine, 11(1), 75, doi: 10.1186/1741-7015-11-75.

Hynynen, S. T., van Stralen, M. M., Sniehotta, F. F., Araújo-Soares, V., Hardeman, W., Chinapaw, M. J. M., ... \& Hankonen, N. (2016). A systematic review of school-based interventions targeting physical activity and sedentary behaviour among older adolescents. International Review of Sport and Exercise Psychology, 9, 22-44, http://dx.doi.org/10.1080/1750984X.2015.1081706.

Johnson, B. T., \& Huedo- Medina, T. B. (2011). Depicting estimates using the intercept in metaregression models: the moving constant technique. Research synthesis methods, 2(3), 204-220, doi: $10.1002 / j \mathrm{jsm} .49$.

Knittle, K., De Gucht, V., Hurkmans, E., Vlieland, T. V., \& Maes, S. (2016). Explaining physical activity maintenance after a theory- based intervention among patients with rheumatoid arthritis: Process evaluation of a randomized controlled trial. Arthritis Care \& Research, 68, 203-210, doi:10.1002/acr.22647.

Knittle, K., Dombrowski, S., Nurmi, J., Haukkala, A., Crutzen, R., \& Hankonen, N. (2015). Identifying intervention techniques associated with increases in intention and motivation for physical activity (CRD42015014922). PROSPERO International Prospective Register of Systematic Reviews. Available from: http://www.crd.york.ac.uk/PROSPERO/display record.php?ID=CRD42015014922 
Knittle, K., Maes, S., \& De Gucht, V. (2010). Psychological interventions for rheumatoid arthritis: Examining the role of self- regulation with a systematic review and meta- analysis of randomized controlled trials. Arthritis Care \& Research, 62, 1460-1472, doi:10.1002/acr.20251.

Knittle, K. (2015). We cannot keep firing blanks - yet another appeal for improved RCT reporting: commentary on Peters, de Bruin and Crutzen. Health Psychology Review, 9, 34-37, doi:10.1080/17437199.2014.900721.

Kohl, L., Crutzen, R., \& De Vries, N. K. (2013). Online prevention aimed at lifestyle behaviours: a systematic review of reviews. Journal of Medical Internet Research, 15, e146, doi:10.2196/jmir.2665.

Kwasnicka, D., Dombrowski, S. U., White, M., \& Sniehotta, F. (2016). Theoretical explanations for maintenance of behaviour change: a systematic review of behaviour theories. Health Psychology Review, 10, 277-296, doi:10.1080/17437199.2016.1151372.

Landis, J. R., Koch, G. G. (1977). The measurement of observer agreement for categorical data. Biometrics, 33, 159-174, doi:10.2307/2529310.

Lipsey M. W., \& Wilson, D. B. (2001). Practical meta-analysis. Thousand Oaks, CA: Sage.

Markland, D., Ryan, R. M., Tobin, V. J., \& Rollnick, S. (2005). Motivational interviewing and selfdetermination theory. Journal of Social and Clinical Psychology, 24, 811-831, https://doi.org/10,1521/jscp.2005.24.6.811.

Markland, D., \& Ingledew, D. K. (2007). The relationships between body mass and body image and relative autonomy for exercise among adolescent males and females. Psychology of Sport and Exercise, 8, 836-853, https://doi.org/10.1016/j.psychsport.2006.11.002.

Markland, D., \& Vansteenkiste, M. (2007). Self-determination theory and motivational interviewing in exercise. In M. S. Hagger \& N. L. D. Chatzisarantis (Eds.), Intrinsic motivation and selfdetermination in exercise and sport (pp. 87-99). Champaign, IL: Human Kinetics.

Marshall, S. J., \& Biddle, S. J. (2001). The transtheoretical model of behavior change: a meta-analysis of applications to physical activity and exercise. Annals of Behavioral Medicine, 23(4), 229246, doi: 10.1207/S15324796ABM2304_2. 
Matthews, C. E., George, S. M., Moore, S. C., Bowles, H. R., Blair, A., Park, Y., ... \& Schatzkin, A. (2012). Amount of time spent in sedentary behaviors and cause-specific mortality in US adults. The American Journal of Clinical Nutrition, 95, 437-445, doi:10.3945/ajen.111.019620.

McCulloch, K. C., Albarracín, D., \& Durantini, M. R. (2008). A door to HIV-prevention interventions: how female-targeted materials can enhance female participation. Journal of Applied Social Psychology, 38, 1211-1229, doi:10.1111/j.1559-1816.2008.00345.x.

McMurran, M., \& Ward, T. (2010). Treatment readiness, treatment engagement and behaviour change. Criminal Behaviour and Mental Health, 20, 75-85, doi:10.1002/cbm 762 .

Michie, S., Abraham, C., Whittington, C., McAteer, J., \& Gupta, S. (2009). Effective techniques in healthy eating and physical activity interventions: a meta-regression. Health Psychology, 28, 690-701, doi:10.1037/a0016136.

Michie, S., \& Prestwich, A. (2010). Are interventions theory-based? Development of a theory coding scheme. Health Psychology, 29, 1-8, doi:10.1037/a0016939.

Michie, S., Ashford, S., Sniehotta, F. F., Dombrowski, S. U., Bishop, A., \& French, D. P. (2011). A refined taxonomy of behaviour change techniques to help people change their physical activity and healthy eating behaviours: the CALO-RE taxonomy. Psychology \& Health, 26, 1479-1498, doi:10.1080/08870446.2010.540664.

Michie, S., Richardson, M., Johnston, M., Abraham, C., Francis, J., Hardeman, W., ... \& Wood, C. E. (2013). The behavior change technique taxonomy (v1) of 93 hierarchically clustered techniques: building an international consensus for the reporting of behavior change interventions, Annals of Behavioral Medicine, 46, 81-95, doi:10.1007/s12160-013-9486-6.

Michie, S., Carey, R. N., Johnston, M., Rothman, A. J., De Bruin, M., Kelly, M. P., \& Connell, L. E. (2016). From theory-inspired to theory-based interventions: A protocol for developing and testing a methodology for linking behaviour change techniques to theoretical mechanisms of action. Annals of Behavioral Medicine, 1-12, doi:10.1007/s12160-016-9816-6. 
Moher, D., Liberati, A., Tetzlaff, J., \& Altman, D. G. (2009). Preferred reporting items for systematic reviews and meta-analyses: the PRISMA statement. Annals of Internal Medicine, 151, 264-269, https://doi.org/10.1371/journal.pmed.1000097.

Morris, S. B., \& DeShon, R. P. (2002). Combining effect size estimates in meta-analysis with repeated measures and independent-groups designs. Psychological Methods, 7, 105-125, doi:10.1037/1082-989X.7.1.105.

Ng, J. Y., Ntoumanis, N., Thøgersen-Ntoumani, C., Deci, E. L., Ryan, R. M., Duda, J. L., \& Williams, G. C. (2012). Self-determination theory applied to health contexts: A meta-analysis. Perspectives on Psychological Science, 7, 325-340, doi:10.1177/1745691612447309.

Nurmi, J., Hagger, M. S., Haukkala, A., Araújo-Soares, V., \& Hankonen, N. (2016). Relations between autonomous motivation and leisure-time physical activity participation: The mediating role of self-regulation techniques. Journal of Sport and Exercise Psychology, 38, 128-137, https://doi.org/10.1123/jsep.2015-0222.

Olander, E. K., Fletcher, H., Williams, S., Atkinson, L., Turner, A., \& French, D. P. (2013). What are the most effective techniques in changing obese individuals' physical activity self-efficacy and behaviour: a systematic review and meta-analysis. International Journal of Behavioral Nutrition and Physical Activity, 10, 29, https://doi.org/10.1186/1479-5868-10-29.

Peters, G. J. Y., de Bruin, M., \& Crutzen, R. (2015). Everything should be as simple as possible, but no simpler: towards a protocol for accumulating evidence regarding the active content of health behaviour change interventions. Health Psychology Review, 9, 1-14, doi:10.1080/17437199.2013.848409.

Presseau, J, Ivers, N. M., Newham, J. J., Knittle, K., Danko, K. J., \& Grimshaw, J. M. (2015). Using a behaviour change techniques taxonomy to identify active ingredients within trials of implementation interventions for diabetes care. Implementation Science, 10, 55, https://doi.org/10.1186/s13012-015-0248-7. 
Prochaska, J. O., \& DiClemente, C. C. (1986). Toward a comprehensive model of change. In W. R. Miller \& N. Heather (Eds.), Treating addictive behaviors: Processes of change (pp. 3-27). New York, NY: Plenum.

Prochaska, J. O., \& Velicer, W. F. (1997). The transtheoretical model of health behavior change. American Journal of Health Promotion, 12, 38-48, https://doi.org/10.4278/0890-1171-12.1.38.

Rhodes, R. E., \& Dickau, L. (2012). Experimental evidence for the intention-behavior relationship in the physical activity domain: A meta-analysis. Health Psychology, 31(6), 724-727, http://dx.doi.org/10.1037/a0027290.

Ruiter, R. A., Abraham, C., \& Kok, G. (2001). Scary warnings and rational precautions. A review of the psychology of fear appeals. Psychology and Health, 16(6), 613-630. https://doi.org/10.1080/08870440108405863

Ryan, R. M. (1995). Psychological needs and the facilitation of integrative processes. Journal of Personality, 63, 397-427, doi:10.1111/j.1467-6494.1995.tb00501.x.

Ryan, R. M., \& Connell, J. P. (1989). Perceived locus of causality and internalization: examining reasons for acting in two domains. Journal of Personality and Social Psychology, 57, 749-761, http://dx.doi.org/10.1037/0022-3514.57.5.749.

Ryan, R. M., \& Deci, E. L. (2000), Self-determination theory and the facilitation of intrinsic motivation, social development, and well-being. American Psychologist, 55, 68-78, http://dx.doj.org/10.1037/0003-066X.55.1.68.

Ryan, R. M., \& Patrick, H. (2009). Self-determination theory and physical activity: The dynamics of motivation in development and wellness. Hellenic Journal of Psychology, 6(2), 107-124.

Schwarzer, R., Lippke, S., \& Luszczynska, A. (2011). Mechanisms of health behavior change in persons with chronic illness or disability: The Health Action Process Approach (HAPA). Rehabilitation Psychology, 56, 161-170, doi:10.1037/a0024509.

Sheeran, P., \& Webb, T. L. (2016). The Intention-Behavior Gap. Social and Personality Psychology Compass, 10, 503-518, doi:10.1111/spc3.12265. 
Stacey, F. G., James, E. L., Chapman, K., Courneya, K. S., \& Lubans, D. R. (2015). A systematic review and meta-analysis of social cognitive theory-based physical activity and/or nutrition behavior change interventions for cancer survivors. Journal of Cancer Survivorship, 9, 305-338, doi:10.1007/s11764-014-0413-z.

Steinmetz, H., Knappstein, M., Ajzen, I., Schmidt, P., \& Kabst, R. (2016). How effective are behayior change interventions based on the Theory of Planned Behavior? A three-level meta-analysis. Zeitschrift Fur Psychologie-Journal of Psychology, 224, 216-233. $\underline{\text { http://dx.doi.org/10.1027/2151-2604/a000255 }}$

Sutton, S. (2008). How does the health action process approach (HAPA) bridge the intention-behavior gap? An examination of the model's causal structure. Applied Psychology, 57, 66-74, doi:10.1111/j.1464-0597.2007.00326.x.

Teixeira, P. J., Carraça, E. V., Markland, D., Silva, M. N., \& Ryan, R. M. (2012). Exercise, physical activity, and self-determination theory: a systematic review. International Journal of Behavioral Nutrition and Physical Activity, 9, 78, https://doi.org/10.1186/1479-5868-9-78.

Teixeira, P., \& Hagger, M. (2016). Motivation and behaviour change techniques based on selfdetermination theory: a consensus analysis. Presented at European Health Psychology Society Annual Conference, Aberdeen, UK; 25 August 2016.

Wasserkampf, A., Silva, M. N., Santos, I. C., Carraça, E. V., Meis, J. J. M., Kremers, S. P. J., \& Teixeira, P. J. (2014). Short-and long-term theory-based predictors of physical activity in women who participated in a weight-management program. Health Education Research, 29, 941-952, doi:10.1093/her/cyu060.

Webb, T., Joseph, J., Yardley, L., \& Michie, S. (2010). Using the internet to promote health behavior change: a systematic review and meta-analysis of the impact of theoretical basis, use of behavior change techniques, and mode of delivery on efficacy. Journal of Medical Internet Research, 12, e4, doi:10.2196/jmir.1376. 
Webb, T. L., \& Sheeran, P. (2006). Does changing behavioral intentions engender behavior change? A meta-analysis of the experimental evidence. Psychological Bulletin, 132, 249-268, doi:10.1037/0033-2909.132.2.249.

Wee, C. C., Davis, R. B., \& Phillips, R. S. (2005). Stage of readiness to control weight and adopt weight control behaviors in primary care. Journal of General Internal Medicine, 20, 410-415, doi:10.1111/j.1525-1497.2005.0074.x.

Williams, S. L., \& French, D. P. (2011). What are the most effective intervention techniques for changing physical activity self-efficacy and physical activity behaviour-and are they the same?. Health Education Research, 26, 308-322, doi:10.1093/her/cyr005.

Wilson, D. B. (2001). Practical Meta-Analysis Effect Size Calculator. Retrieved from http://www.campbellcollaboration.org/escalc/html/EffectSizeCalculator-Home.php. 
Table 1 - Effect sizes obtained from comparative subgroups analyses of BCTs which revealed a significant association with at least one motivational construct.

\begin{tabular}{|c|c|c|c|}
\hline Moderator - Interventions containing the following & Intention $(k=77)$ & Stage of Change $(k=96)$ & Autonomous Motivation $(k=34)$ \\
\hline BCT 1.1 - Behavioral Goal Setting & $0.12(0.00,0.24) ; 42$ & $0.20(0.04,0.36) ; 54$ & $0.14(-0.02,0.29) ; 14$ \\
\hline BCT 1.2 - Problem Solving & $0.12(-0.01,0.25) ; 21$ & 0.33 & $0.08(-0.05,0.22) ; 10^{\mathrm{a}}$ \\
\hline BCT 1.4 - Action Planning & $0.08(-0.05,0.21) ; 29$ & $0.27(0.0$ & $0.08(-0.05,0.22) ; 10^{\mathrm{a}}$ \\
\hline BCT 2.2 - Feedback on Behavior & $0.12(-0.02,0.26) ; 12$ & 19 & $0.04(-0.08,0.17) ; 9$ \\
\hline BCT 2.3 - Self-monitoring of behavior & $0.24(0.07,0.41) ; 17$ & $0.46) ; 34$ & $0.06(-0.09,0.20) ; 9$ \\
\hline BCT 3.2 - Practical social support & & $(-0.46,-0.09) ; 3$ & $\mathrm{~N} / \mathrm{A}$ \\
\hline BCT 4.1 - Instruction on how to perform behavior & $.01,0$ & $0.43(0.11,0.75) ; 18$ & $0.19(-0.02,0.40) ; 10$ \\
\hline BCT 5.3 - Info about social / environmental consequences & $15,0.46) ; 15$ & $-0.16(-0.39,0.08) ; 11$ & $-0.13(-0.32,0.07) ; 5$ \\
\hline BCT 6.1 - Demonstration of behavior & $025)$. & $0.39(0.12,0.66) ; 14$ & $0.19(0.03,0.35) ; 11$ \\
\hline BCT 8.1 - Behavioral practice & $02,0.42) ; 10$ & $0.46(0.05,0.86) ; 11$ & $0.21(-0.02,0.45) ; 9$ \\
\hline BCT 8.7 - Graded tasks & $\mathrm{N} / \mathrm{A}$ & $0.44(0.20,0.68) ; 21$ & $0.08(-0.06,0.21) ; 8$ \\
\hline BCT 10.7 - Self-incentive & $\mathrm{N} / \mathrm{A}$ & $0.5(0.07,0.92) ; 5$ & $\mathrm{~N} / \mathrm{A}$ \\
\hline BCT 12.2 - Restructuring the social e & $\mathrm{N} / \mathrm{A}$ & $-0.23(-0.42,-0.03) ; 6$ & $0.14(-0.15,0.42) ; 3$ \\
\hline BCT 12.5 - Adding objects to the environment & $\mathrm{N} / \mathrm{A}$ & $0.42(0.20,0.64) ; 3$ & $0.08(-0.10,0.25) ; 6$ \\
\hline
\end{tabular}


BCT 12.6 - Body changes

BCT 15.1 - Verbal persuasion about capability

BCT 15.2 - Mental rehearsal of successful performance

BCT 17.1 - Offer pedometer or wearable

Control theory techniques

BCT $2.3+$ BCT $1.1,1.2,1.4$ or 2.2
N/A

$0.06(-0.11,0.23) ; 5$

$0.46(0.11,0.81) ; 6$

$\mathrm{N} / \mathrm{A}$

$0.24(0.07,0.41) ; 17$
$-0.47(-0.69,-0.24) ; 4$

$-0.21(-0.38,-0.04) ; 4$

$0.05(-0.22,0.32) ; 3$

Note. Data shown are Effect size (LL 95\% CI, UL 95\% CI); number of study arms reporting on this outcome in which BCT was present. Effect sizes are the difference between effect sizes from interventions which included a BCT and those which did not. Results in bold indicate that the $95 \%$ CI for the difference does not include zero. Positive effect sizes represent beneficial effects on motivational outcomes. Comparisons with the same superscript letters compared the same groups of interventions. N/A = No comparison possible because fewer than three interventions reporting on the outcome included the BCT in question. 
Table 2 - Effect sizes obtained from comparative subgroups analyses of moderator variables which revealed a significant association with at least one motivational construct.

\begin{tabular}{|c|c|c|c|}
\hline Moderator & Intention $(k=77)$ & Stage of Change $(k=96)$ & nomous Motivation $(k=34)$ \\
\hline Components delivered face-to-face & $0.18(0.06,0.31) ; 34$ & $0.33(0.17,0$. & $0.19(0.04,0.34) ; 21$ \\
\hline Components delivered in a group & $0.17(0.02,0.32) ; 20$ & $0.22(C$ & $-0.05(-0.26,0.17) ; 8$ \\
\hline Components delivered via telephone & $-0.17(-0.39,0.05) ; 4$ & 15 & $0.14(0.01,0.27) ; 4$ \\
\hline Components delivered via postal mail & $-0.24(-0.43,-0.04) ; 9$ & ); 9 & $-0.27(-0.48,-0.06) ; 3$ \\
\hline Components delivered in gym & N/A & 7); 7 & $0.22(0.05,0.40) ; 11$ \\
\hline Components delivered in a university & $0.31(0.08$ & $0.09(-0.34,0.51) ; 6$ & N/A \\
\hline Components delivered by a gym worker or trainer & 0.03 & $0.54(0.34,0.74) ; 18$ & $0.25(0.10,0.41) ; 14$ \\
\hline Components delivered by a researcher & 16 & $-0.11(-0.37,0.15) ; 11$ & N/A \\
\hline Components delivered by a physiotherapist & 4 & $-0.34(-0.48,-0.19) ; 3$ & $\mathrm{~N} / \mathrm{A}$ \\
\hline Components delivered by a peer facilitator & N/A & $-0.18(-0.36,-0.01) ; 3$ & $\mathrm{~N} / \mathrm{A}$ \\
\hline Some intervention component explicitly targeted & $0.10(-0.09,0.30) ; 6$ & $0.31(0.04,0.58) ; 18$ & $-0.01(-0.12,0.11) ; 4$ \\
\hline $\begin{array}{l}\text { Some intervention component explicitly targeted } v \\
\text { the theory of planned behavior, reasoned action ap }\end{array}$ & $0.25(0.08,0.42) ; 10$ & $0.28(0.12,0.44) ; 5$ & N/A \\
\hline
\end{tabular}


Delivered to sedentary individuals

Delivered to overweight individuals
$0.09(-0.05,0.22) ; 36$

$-0.12(-0.29,0.05) ; 8$
$0.48(0.33,0.64) ; 51$

$0.67(0.34-1.00) ; 9$
$-0.12(-0.28,0.04) ; 20$

Note. Data shown are Effect size (LL 95\% CI, UL 95\% CI); number of study arms reporting on this outcome in which moderator was present. Effect sizes are the difference between interventions which included a component and those which did not. Positive effect sizes represent beneficial effects on motivational outcomes. Results in bold indicate that the $95 \% \mathrm{CI}$ for the difference does not include zero. N/A = No comparison possible because fewer than three arms reporting on the outcome included the BCT/component in question. * = item five from Michie \& Prestwich (2010), "Theory/predictors used to select/develop intervention techniques." 
Figure 1. PRISMA Flow Diagram of Search Procedures

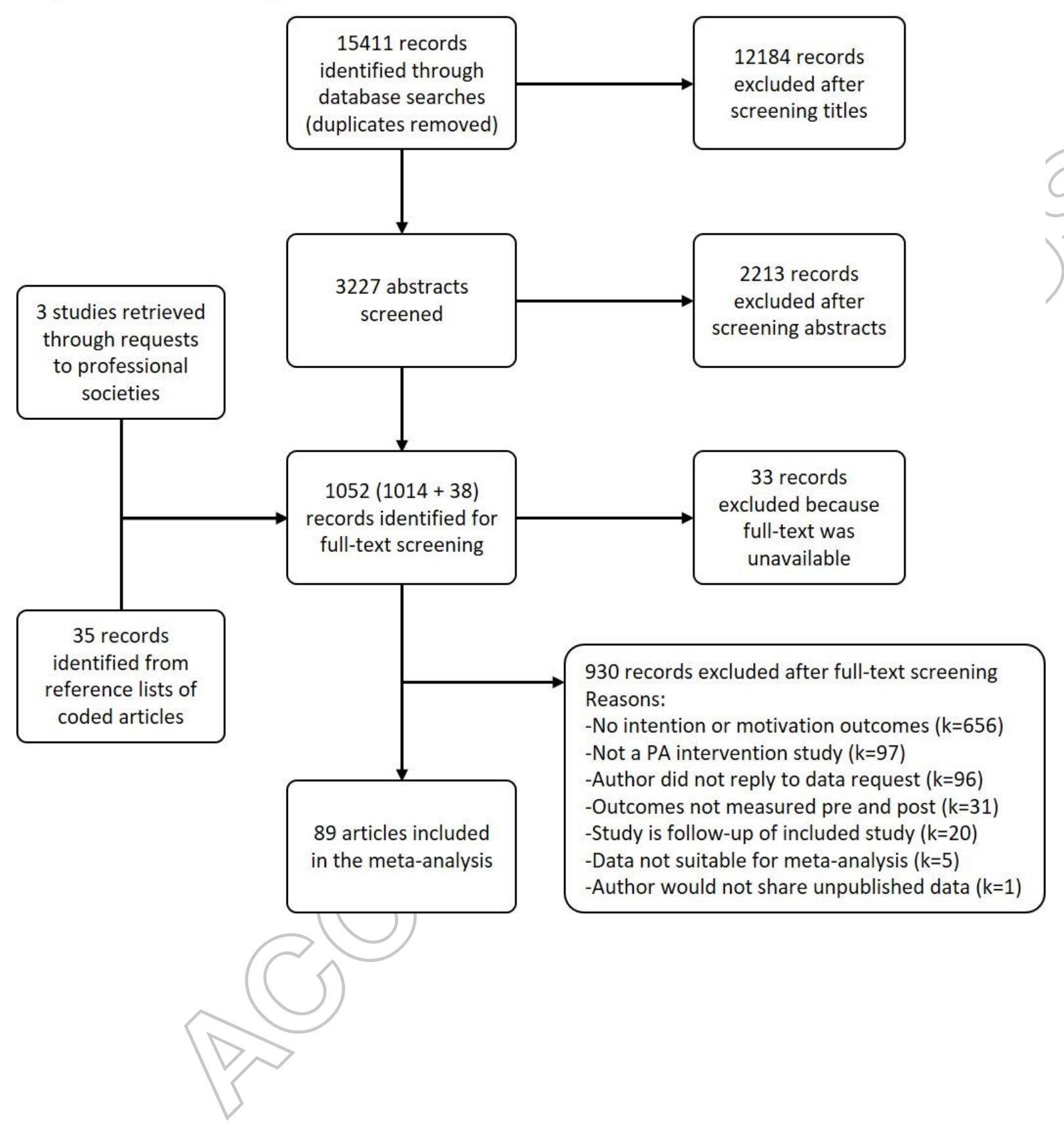

